\title{
Research highlights about contributions on cancer published in Electronic Physician Journal between 2009 and 2015
}

\section{Abdeljalil Khelassi}

Associate Professor, Abou Bekr Belkaid University of Tlemcen, Algeria

Type of article: Editorial report

\begin{abstract}
This article aims to highlight the important research work on cancer published in Electronic Physician Journal. The journal has published 18 articles concerning cancer research, i.e., two review articles, two case reports, and 14 original articles from 2009 through 2015. The types of cancer are breast cancer, prostate cancer, ovarian cancer, leukemia, cervical cancer, rectal cancer, and papillary thyroid Cancer. In addition, the articles have addressed several aspects of cancer, including prevention, diagnosis, follow-up, and therapy.

Keywords: Neoplasms, Prevention and control, Diagnosis, Therapeutics, Publishing
\end{abstract}

\section{Introduction}

Cancer is still a crucial epidemic disease, which requires more effort and research findings. From prevention and prognosis to surgery and treatment, all stages are important parts of the research axes. Electronic Physician Journal aims to publish high-quality research findings in medicine. It is an international, peer-reviewed, open-access journal, and gradually, it has become well indexed by several master lists of international research organizations and academic libraries (Figures 1, 2). In this editorial, we introduce the published articles relative to cancer, and we highlight the findings of these research efforts.

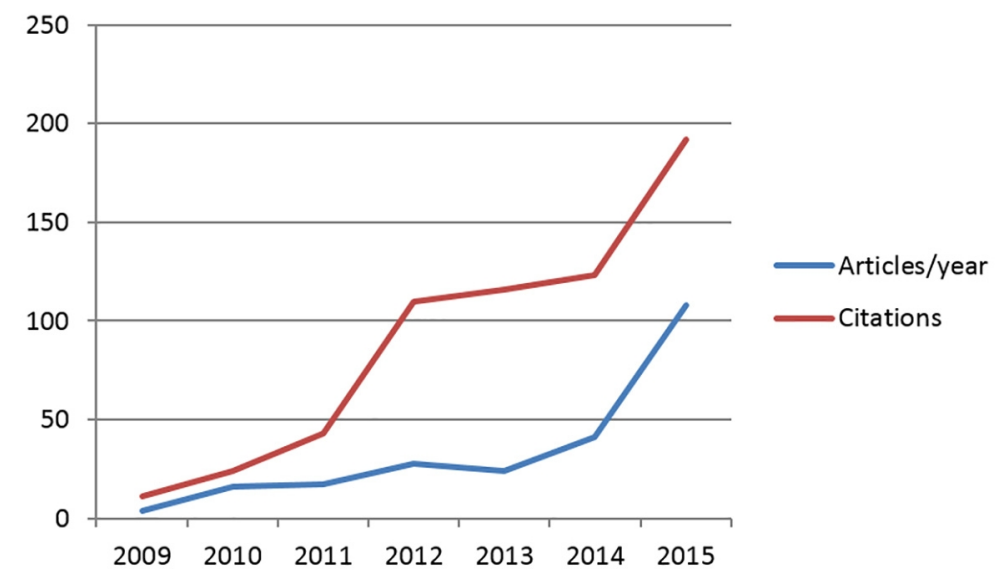

Figure 1. Visibility of Electronic Physician Journal on Google-scholar measured by "Harzing's Publish or Perish, v4.15" software

\section{Corresponding author:}

Associate Professor Dr. Abdeljalil Khelassi, Abou Bekr Belkaid University of Tlemcen, Algeria.

Email: KHELASSI.A@gmail.com

Received: May 15, 2016, Accepted: July 12, 2016, Published: December 2016

(C) 2016 The Authors. This is an open access article under the terms of the Creative Commons Attribution-NonCommercialNoDerivs License, which permits use and distribution in any medium, provided the original work is properly cited, the use is non-commercial and no modifications or adaptations are made. 


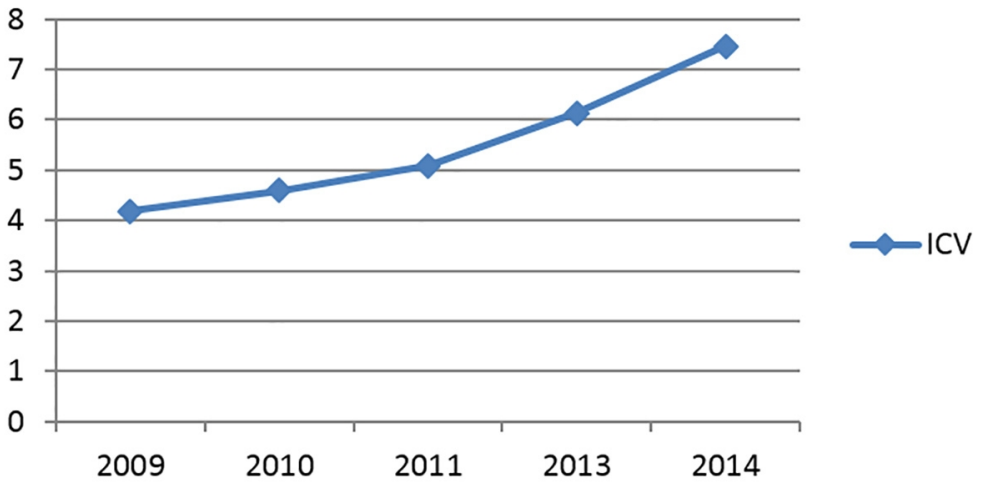

Figure 2. The Index Copernicus Value (ICV) of Electronic Physician Journal [2009-2014]

\section{Research highlights}

Tables 1 and 2 present the types of cancers and their stages that the published articles have addressed.

\subsection{Original articles}

In this section, we present the inferred conclusions of the highlighted original articles concerning cancer:

1) Quita and Balbaid (2015) presented a clinical trial about the effect of lemon fruit extract against the toxicity of cyclophosphamide (CP) used in the chemotherapy against cancer. The conclusion of this clinical trial was that the lemon fruit extract had a protective effect against CP-induced intestinal and pancreatic injury (1).

2) Karimi-Zarchi et al. (2015) presented a survey about the influence of the stage of diagnosed ovarian cancer on the mortality of patients. The conclusion reported in this study was that the survival of patients with epithelial ovarian cancer was significantly associated with the stage of the disease. They also reported that serous adenocarcinoma had the best prognosis among the pathologies studied. Therefore, early detection of ovarian cancer can substantially increase the survival rate (2).

3) Haghighi, S. T. et al. (2015) presented a sociological study about the influence of the level of patients' literacy on the health promotion of breast cancer patients. The conclusion of this research work was that the promotion of the public health is relative to the publics' awareness of breast cancer as measured by the patients' level of health literacy (3).

4) Rakhsha, A. et al. (2015) presented results of seven years' experience of follow-up with prostate specific antigens (PSA). They concluded that the PSA was the strongest predictor of biochemical progression and survival in patients with prostate cancer who were treated with definitive external beam radiation therapy (4).

5) Mehrabi, E. et al. (2015) presented a qualitative research study about the influence of the experiences of 18 women with breast cancer concerning post-treatment growth (PTG). The authors strongly recommended that health care professionals should design robust and timely intervention programs to decrease the rate of PTG among breast cancer survivors and to reduce their distress as the result of their cancer diagnosis (5).

6) Mofid, B. et al. (2015) presented a study about the protective effect of the mucilaginous herb (Malva) against the urinary toxicity generated by external beam radiation therapy (EBRT). By examining 60 cases with prostate cancer and comparing the effect of Malva compared with a placebo after EBRT, the authors reported positive results (6).

7) Jeihooni and Kashfi (2015) presented a survey about the prostate cancer screening behaviors of 400 men over 50 in Faza, Iran. They highlighted the most important factors that influenced the prostate cancer screening behavior, such as the knowledge, attitudes, and beliefs of individuals, as well as other reinforcing factors (7).

8) Houshyari, M. et al. (2015) presented a comparative study of two breast cancer treatment techniques (single anterior posterior (AP) and two anterior and posterior opposed (AP/PA)). After examining 40 cases, they concluded that the AP/PA treatment technique was a more suitable choice of treatment than the AP field, especially for overweight and obese breast cancer patients. However, in the AP/PA technique, the use of a single-photon, low-energy (6 MV) radiotherapy caused more hot spots than usual (8).

9) Khelassi, A. (2014) presented a component for an explanation-aware of breast cancer prognosis supported by IK-DCBRC, which is a complex, computer-aided diagnosis system. The presented explanation agent 
aims to reduce uncertainty and risks by ensuring the transparency of the automatic diagnosis of breast cancer supported by IK-DCBRC. The component sensitizes physicians about the uncertainty of their decisions, which decreased doubts and helped detect some conflicts (9).

10) Karimi-zarchi M. et al. (2015) presented a comparative survey between the colposcopy and pap smear techniques for the detection of cervical cancer. According to this study, the accuracy, sensitivity, specificity, and negative predictive values of colposcopy were higher than those for the Pap smear in detecting high-grade, cervical, pre-malignant lesions (cervical intraepithelial neoplasia: CIN $\geqslant 2$ ) (10).

11) Karimi-Zarchi M. et al. (2015) conducted research to evaluate pelvic masses and detect malignancies with the ultrasound technique by considering the Risk Management Index (11).

12) Mona M Zoheiry et al. (2015) presented a possibility for malignancy prediction in HCV-genotype by observing TGF- $\beta 1 /$ BMP-7 ratio and CTGF levels, which reflect the rate of EMT and provide information about fibrogenic activity, in association with YKL-40 (12).

13) Sepideh Banava, et al. (2015) presented a study about the status of oral and saliva conditions of patients undergoing chemotherapy with and without application of CPP-ACPF. The study indicated that there was a positive effect of CPP-ACPF in the salivary status of patients undergoing chemotherapy (13).

14) Ali Zedan and Tareq Salah (2015) presented a study that aimed to determine the morbidity, mortality, survival outcome, and local failure after total mesorectal excision (TME) in the surgical treatment of rectal cancer. After clinical trials, the authors reported an enhanced role and efficiency of the TME technique in rectal cancer surgery. They also improved the safety of TME with neoadjuvant chemo-radiotherapy in local control and long-term survival and maintenance of a good quality of life (14).

\subsection{Review articles}

In this section, we present the inferred conclusions of the highlighted review articles concerning cancer:

1) Hashemi, S. H. B. et al. (2014) reviewed the most important lifestyle factors for the prevention of breast cancer in women. In this research, they highlighted the impact of diet, physical activity, smoking, alcohol use, and vitamin and mineral use on breast cancer. The lifestyle factors of women with breast cancer were from the most prestigious research articles that studied the lifestyles of women who were undergoing treatment for breast cancer (15).

2) Esmat Mehrabi et al. (2015) presented a systematic review about breast cancer. This article indexes the existing published articles concerning the coping responses of breast cancer patients. This research effort was an essential follow-up of this epidemic illness (16).

\subsection{Case reports}

1) Samadi, S. et al. (2015) presented a pediatric case report about a 2.5 -year-old male patient with acute lymphoblastic leukemia. The report highlights an unexpectedly pulseless radial artery, which was preliminarily and unduly attributed to the anesthesia before the radiotherapy. Their conclusions suggested that an accurate history and physical examination before the initiation of anesthesia or sedation might be useful in preventing the mismanagement of procedures in treating pediatric cancer (17).

2) Ranjbar, S. H., and Jahani, S. A. (2012) presented a case report about papillary thyroid cancer in an 80year-old man. The aim of this report was to highlight the milliary lung metastasis in this case (18).

Table 1. Contributions by the type of cancer*

\begin{tabular}{|l|l|}
\hline Cancer type & Contributions \\
\hline Breast cancer & $(3),(5),(9),(10),(11),(16)$ \\
\hline Prostate cancer & $(4),(8)$ \\
\hline Ovarian cancer & $(2)$ \\
\hline Leukemia & $(7)$ \\
\hline Papillary Thyroid Cancer & $(12)$ \\
\hline Cervical cancer & $(13)$ \\
\hline Rectal cancer & $(18)$ \\
\hline All types of cancer & $(1),(6),(14),(15),(17)$ \\
\hline
\end{tabular}

*Citation policy: The citations to mentioned articles in this editorial are removed to prevent the self-citations to our journal. The in-text citations to the number of mentioned articles (and tables) are not included in reference section. 
Table 2. Contributions by the stage of the cancer*

\begin{tabular}{|l|l|}
\hline Cancer stages & Contributions \\
\hline Prevention & $(11)$ \\
\hline Diagnosis & $(2),(8),(10),(12),(13),(14),(15)$ \\
\hline Follow-up and Therapy & $(1),(3),(4),(5),(6),(7),(9),(12),(16),(17),(18)$ \\
\hline
\end{tabular}

*Citation policy: The citations to mentioned articles in this editorial are removed to prevent the self-citations to our journal. The in-text citations to the number of mentioned articles (and tables) are not included in reference section.

\section{Conclusions}

Although cancer represents a primordial epidemic disease, some weaknesses can be identified in all stages. To date, Electronic Physician's publications concerning cancer have been few, representing just $8 \%$ of the total contributions (i.e., only 18 out of 238 articles). In this editorial, we have provided the cancer researchers' community an index about the published contributions in Electronic Physician journal in the period from 2009 through 2015. The research about cancer is a multi-disciplinary domain, and Electronic Physician Journal solicits all communities to publish their findings. It is an honor to participate in this noble work.

\section{Conflict of Interest:}

There is no conflict of interest to be declared. 